\title{
Calculation of anharmonic OH phonon dispersion curves for the $\mathrm{Mg}(\mathrm{OH})_{2}$ crystal
}

Cite as: J. Chem. Phys. 133, 034120 (2010); https://doi.org/10.1063/1.3458001

Submitted: 19 March 2010 . Accepted: 08 June 2010 . Published Online: 21 July 2010

Pavlin D. Mitev, Kersti Hermansson, and Wim J. Briels

\section{ARTICLES YOU MAY BE INTERESTED IN}

Anharmonic $\mathrm{OH}$ vibrations in $\mathrm{Mg}(\mathrm{OH})_{2}$ (brucite): Two-dimensional calculations and crystalinduced blueshift

The Journal of Chemical Physics 131, 244517 (2009); https://doi.org/10.1063/1.3266507

Comparing van der Waals DFT methods for water on $\mathrm{NaCl}(001)$ and $\mathrm{MgO}(001)$

The Journal of Chemical Physics 146, 064703 (2017); https://doi.org/10.1063/1.4971790

Perspective: Machine learning potentials for atomistic simulations

The Journal of Chemical Physics 145, 170901 (2016); https://doi.org/10.1063/1.4966192

The Journal

of Chemical Physics

The Emerging Investigators Special Collection and Awards Recognizing the excellent work of early career researchers! 


\title{
Calculation of anharmonic $\mathrm{OH}$ phonon dispersion curves for the $\mathrm{Mg}(\mathrm{OH})_{2}$ crystal
}

\author{
Pavlin D. Mitev, ${ }^{1, a)}$ Kersti Hermansson, ${ }^{1}$ and Wim J. Briels ${ }^{2}$ \\ ${ }^{1}$ Materials Chemistry, The Ångström Laboratory, Uppsala University, Box 538, Uppsala S-75121, Sweden \\ and Theoretical Chemistry, School of Biotechnology, Royal Institute of Technology, \\ Stockholm 10691, Sweden \\ ${ }^{2}$ Computational Biophysics, Twente University, P.O. Box 217, AE Enschede 7500, The Netherlands
}

(Received 19 March 2010; accepted 8 June 2010; published online 21 July 2010)

\begin{abstract}
Anharmonic OH phonon dispersion curves have been calculated for the $\mathrm{Mg}(\mathrm{OH})_{2}$ crystal. A crystal Hamiltonian was set up for the vibrational problem, where the coordinates consists of the bond lengths of two hydroxide ions in the central unit cell. Its two-dimensional potential energy surface was constructed from first principle calculations within the density functional theory approximation. Dispersion curves were calculated by diagonalizing the Hamiltonian in a basis of singly excited crystal functions. The single particle functions used to construct the crystal states were taken from a Morse oscillator basis set. These well chosen functions made it possible to restrict calculations to include only very few functions, which greatly contributed to a transparent presentation of the underlying theory. All calculations could be done analytically except for the calculation of a few integrals. We have compared our results with those of a series of harmonic lattice dynamics calculations and have found that the anharmonicity shifts the IR and Raman dispersion curves downward appreciably and slightly changes the energy differences between both curves. From an analysis of the harmonic results we conclude that incorporating the coupling between $\mathrm{OH}$ stretching motion and the motion of their centers of mass will appreciably change the overall features of the dispersion curves. Extension of the anharmonic model along these lines will cause no problem to the theoretical approach presented in this paper. () 2010 American Institute of Physics. [doi:10.1063/1.3458001]
\end{abstract}

\section{INTRODUCTION}

Layered hydroxides have important applications as catalysts in chemical, environmental, and biomimetic processes and as ion exchangers and sorbents (see for example a recent review on layered hydroxides and clays by Civalleri et al.). ${ }^{1}$ The mineral brucite, $\operatorname{Mg}(\mathrm{OH})_{2}$, is a prototype among the layered hydroxides. The crystal structure is made up of infinite $(\mathrm{HO})^{-}-\mathrm{Mg}^{2+}-(\mathrm{OH})^{-}$layers repeated periodically along the $\vec{c}$-axis, or equivalently it can be described as two antiparallel sublattices of hydroxide ions stacked between two $\mathrm{Mg}^{2+}$ layers and repeated along the $\vec{c}$-axis (Fig. 1).

An accurate quantum-mechanical calculation of anharmonic $\mathrm{OH}$ vibrational frequencies (in any crystal) requires three ingredients: a potential energy surface (PES) of satisfactory quality, an appropriate Hamiltonian for the vibrational problem, and an adequate method to solve the vibrational Schrödinger equation. One popular method to study anharmonic effects in the dynamics of crystals is called mode following, or the method of frozen phonons. It consists of first performing a harmonic calculation and next calculating the dynamics of the crystal by moving all atoms along one particular phonon coordinate while all others are kept equal to zero. In more detail this means that the potential energy of the crystal is expanded to second order in the atomic displacements around their equilibrium positions and

${ }^{a)}$ Electronic mail: pmitev@mkem.uu.se. that next the kinetic and potential energies are diagonalized simultaneously by means of a simple linear coordinate transformation. This can be done because after mass weighing the coordinates, the kinetic energy is just a fully isotropic quadratic form that will remain diagonal under any orthogonal linear transformation of the coordinates. After the diagonalization, the Hamiltonian is separated in the sense that it is a sum of independent harmonic oscillator Hamiltonians, each depending on just one of the coordinates. In the next step of the mode-following approach it is assumed that the Hamiltonian remains partially separated in terms of the harmonic oscillator coordinates, even after anharmonic corrections have been added to the potential energy. In case one is interested in one particular mode the anharmonic Hamiltonian is assumed to consist of two parts: one which depends only on the chosen phonon coordinate and one which depends in whichever complex way on the remaining phonon coordinates. In pictorial terms this means that one walks along the potential energy surface in a particular direction and that the potential energy surface perpendicular to that direction looks exactly the same at each point along the selected coordinate. If this condition is not met, mode following is a dangerous exercise which may have no direct relation to reality.

The mode-following approach has often been used in the literature to calculate anharmonic frequencies of phonon vibrations whose main contributions come from $\mathrm{OH}$ stretching coordinates, and for which it is assumed that they are decoupled from all other degrees of freedom. The anharmonic fre- 
(a)

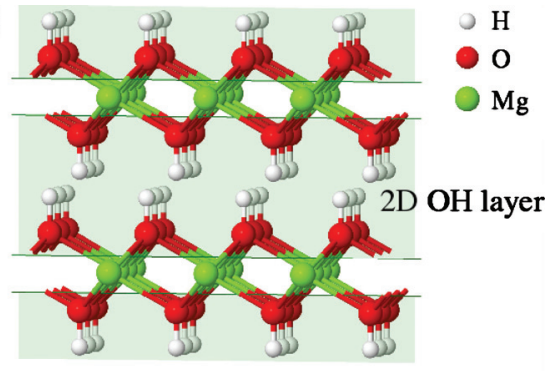

(b)

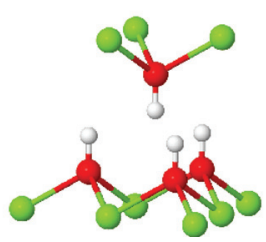

FIG. 1. (a) The structure of the $\mathrm{Mg}(\mathrm{OH})_{2}$ crystal. (b) A crystal fragment visualizing the three nearest neighbor $\mathrm{OH}^{-}$ions surrounding an $\mathrm{OH}^{-}$ion in the crystal.

quency for the Raman-active mode in $\operatorname{Mg}(\mathrm{OH})_{2}$, for example, has been calculated in this way from potential energy curves derived from quantum-mechanical calculations in Refs. 2-7; the stretching coordinates of all $\mathrm{OH}$ groups (both $\mathrm{O}$ and $\mathrm{H}$, or just $\mathrm{H}$ ) in the two hydroxide sublattices were varied by the same amount (while keeping constant all other nuclear coordinates at their equilibrium values). This is tantamount to exploring the southwest-northeast diagonal of the full two-dimensional $\mathrm{OH}$ potential energy surface in Fig. 2.

The mode-following approach is questionable for crystals with (close) degeneracy between vibrations involving

\section{2-D PES for $\mathrm{OH}$ vibrations in the} $\mathrm{Mg}(\mathrm{OH})_{2}$ crystal from DFT calculations

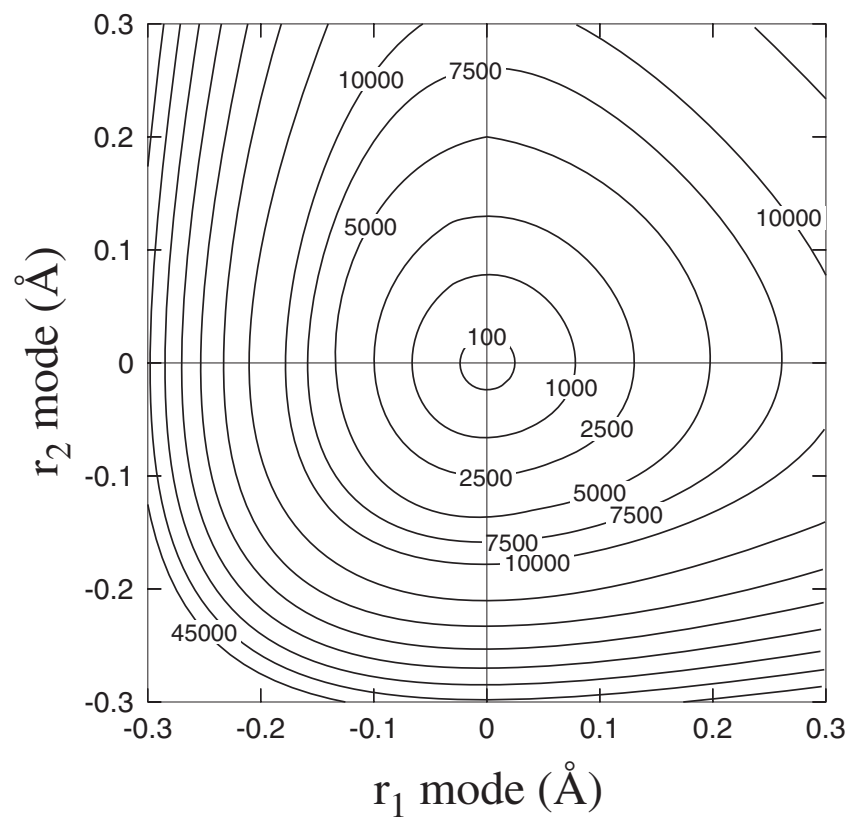

FIG. 2. 2D potential energy surface, $V\left(x_{1}, x_{2}\right)$ for brucite taken from our DFT calculations in Ref. 8. The energy values are for the total energy for the $\mathrm{Mg}(\mathrm{OH})_{2}$ unit cell (in $\left.\mathrm{cm}^{-1}\right)$ in the plane spanned by the $r_{1}=r(\mathrm{OH} 1)$ $-r_{e}(\mathrm{OH} 1)$ and $r_{2}=r(\mathrm{OH} 2)-r_{e}(\mathrm{OH} 2)$ displacement coordinates. oscillators which interact appreciably among each other, as is the case for the $\mathrm{OH}$ groups in brucite. In order to investigate this problem, a Hamiltonian pertaining to two $\mathrm{OH}$ oscillators was diagonalized in Ref. 8, and the results were compared with those obtained with a frozen phonon approach as an approximate treatment of the same Hamiltonian. It was clear that the frozen phonon model of the Raman mode was inadequate and, for example, underestimated the anharmonicity contribution by almost $50 \%$.

In the present paper, we continue the work presented in Ref. 8, where the Hamiltonian including the stretching energies of the two antiparallel $\mathrm{OH}$ groups in the unit cell was diagonalized in a basis of product functions of harmonic oscillator functions. Given the fact that the $\mathrm{OH}$ stretching mode is already anharmonic in the isolated $\mathrm{OH}^{-}$ion, it seems better to use a Morse rather than a harmonic oscillator basis. When using Morse oscillator functions we will find that fully converged results can be obtained by restricting the single particle basis to the Morse oscillator ground and first excited states. This makes it possible to obtain the full solution analytically, apart of course from the calculation of some integrals, and to develop some intuitive feeling for the results. A second result of this paper is that we extend the Hamiltonian presented in Ref. 8, such that it can be used to calculate the dynamics of all $\mathrm{OH}$ groups in the crystal, including the corresponding anharmonic dispersion curves. Moreover, we derive expressions to calculate IR and Raman intensities, which allow us to compare our results with experimental observations.

To the best of our knowledge this is the first time that anharmonic vibrations in an inorganic crystal have been described in great detail. In case our crystal Hamiltonian is restricted such that each $\mathrm{OH}$ group stretches in exactly the same way as the corresponding one in the central unit cell, the approach becomes equivalent to that presented in Ref. 8 .

\section{THEORY-SOLUTION OF A CRYSTAL VIBRATIONAL HAMILTONIAN}

In this section we will describe all theoretical concepts of our vibrational model in detail and provide the equations needed to perform the calculations of the dispersion curves for the anharmonic vibrations. First, in Sec. II A, we will extend the Hamiltonian presented in Ref. 8 for use in calculations of the dynamics of the full crystal (all $N$ unit cells). In Sec. II B we will describe the methods that we use to diagonalize the full Hamiltonian to obtain all $\mathrm{OH}$ stretching vibrations of the crystal. Finally, in Sec. II C, we briefly describe a calculation of the selection rules for IR and Raman experiments allowing us to compare the frequencies that we calculate to particular experimental results.

We now briefly describe in words how the Hamiltonian describing the motions of all $\mathrm{OH}$ groups in the crystal will be diagonalized. The corresponding equations will be given below. The method that we use to diagonalize the Hamiltonian has previously been used to study vibrational motions in molecular crystals of $\mathrm{N}_{2}$ and $\mathrm{O}_{2}$ molecules Refs. 9-14. In these cases, rather extended calculations were necessary to obtain sufficient accuracy, which have concealed the simplicity of the method to a large extent. In the present application cou- 
TABLE I. The first three Morse oscillator functions.

\begin{tabular}{lrl}
\hline \hline$|0\rangle=N_{0} e^{-\alpha y} y^{\alpha-1 / 2}$ & $N_{0}=\sqrt{\frac{a(2 \alpha-1)}{\Gamma(2 \alpha)}(2 \alpha)^{\alpha-1 / 2}} \quad \varepsilon_{0}=\frac{1}{2} \hbar \omega-\frac{1}{4} \frac{\hbar^{2} a^{2}}{2 \mu}$ \\
$|1\rangle=N_{1} e^{-\alpha y} y^{\alpha-3 / 2}(2 \alpha-2-2 \alpha y)$ & $N_{1}=\sqrt{\frac{a(2 \alpha-3)}{\Gamma(2 \alpha-1)}}(2 \alpha)^{\alpha-3 / 2} \quad \varepsilon_{1}=\frac{3}{2} \hbar \omega-\frac{9}{4} \frac{\hbar^{2} a^{2}}{2 \mu}$ \\
$|2\rangle=N_{2} e^{-\alpha y} y^{\alpha-5 / 2} 1 / 2\left[(2 \alpha-3)(2 \alpha-4)-2(2 \alpha-3) 2 \alpha y+4 \alpha^{2} y^{2}\right]$ & $N_{2}=\sqrt{\frac{2 a(2 \alpha-5)}{\Gamma(2 \alpha-2)}}(2 \alpha)^{\alpha-5 / 2} \quad \varepsilon_{2}=\frac{5}{2} \hbar \omega-\frac{25}{4} \frac{\hbar^{2} a^{2}}{2 \mu}$ \\
$\alpha=\sqrt{\frac{2 \mu D}{\hbar^{2} a^{2}}}$ & $y=e^{-a x}$ & $\omega=\sqrt{\frac{2 D a^{2}}{\mu}}$
\end{tabular}

pling terms between the stretching vibrations of the $\mathrm{OH}$ groups are rather weak such that all equations may be presented in a particularly transparent way. We therefore consider it useful to present the theory with most of its details rather than to just refer to the literature.

In a first step we calculate a set of single particle states which will allow us to make use of the Brillouin theorem later on. To find this set of functions we temporarily treat the crystal as an Einstein crystal. Each particle moves independently from all other particles in the average potential or mean field. ${ }^{15}$ The average field felt by a particular particle is calculated by averaging its interaction energies with the surrounding particles over the mean-field ground states of the surrounding particles. The corresponding Schrodinger equations are called mean-field equations and their solution is iterative by nature. The resulting solutions are called single particle mean-field states. In the present application there is just one set of single particle mean-field states since all particles are identical by symmetry.

One way of arriving at the mean-field equations is by constructing a mean-field crystal state as a product of single particle mean-field states, one for every $\mathrm{OH}$ group in the crystal, and then minimizing the expectation value of the crystal Hamiltonian in this crystal state by varying the single particle states. As a consequence, making changes at just one point in the crystal cannot lower the energy of the crystal ground state anymore. Stated differently, this means that the matrix element of the crystal Hamiltonian between the ground state and any crystal state obtained by changing the ground state at just one point will be zero. This result is called the Brillouin theorem, which we will use below when we derive the final expressions to calculate the vibration frequencies.

Details about the mean-field calculation may be found in Appendix A. It so turns out that the mean-field states do not differ very much from the Morse oscillator functions introduced as a basis for all our calculations (the first three are given in Table I). Therefore we will present all further theoretical details using Morse oscillator functions, even when in that case the Brillouin theorem does not apply exactly. The correct expressions may be obtained from the ones presented below by replacing all Morse oscillator functions and their energies by the corresponding mean-field states and energies.

In a second step the exact crystal states will be written as linear combinations of certain crystal states described below.
This amounts to diagonalizing the crystal Hamiltonian in this particular basis of crystal states. In previous application of the method described here, it turned out that to a good approximation the crystal basis may be restricted to the state $|\Phi\rangle$, which is a product of single particle ground-state Morse functions and the $2 N$ states obtained by exciting the single particle Morse ground state to the single particle first excited Morse state at just one position in the crystal. As was mentioned above, in the more accurate approximation all single particle Morse states must be replaced by single particle mean-field states. The Brillouin theorem then states that the Hamiltonian is block diagonal, a $1 \times 1$ block containing the crystal ground state energy, and a $2 N \times 2 N$ block containing the matrix elements of the Hamiltonian between the various singly excited crystal states. The diagonalization of the latter block will turn out to be an easy task once the crystal states have been adapted to the translational symmetry of the crystal.

\section{A. The crystal Hamiltonian}

Harmonic phonon calculations have shown that to a good approximation the stretching vibrations of the two $\mathrm{OH}$ groups in brucite are uncoupled from the other degrees of freedom. Assuming this to be true more generally, i.e., also when anharmonic contributions to the potential energy are taken into account, a Hamiltonian may be written down with only two degrees of freedom per unit cell. Assuming that the potential energy may be well approximated by a sum of pair contributions we write

$$
\begin{aligned}
& H=\sum_{P} h\left(x_{P}\right)+\frac{1}{2} \sum_{P} \sum_{P^{\prime}} w_{P, P^{\prime}}\left(x_{P}, x_{P^{\prime}}\right), \\
& h(x)=-\frac{\hbar^{2}}{2 \mu} \frac{\partial^{2}}{\partial x^{2}}+v(x) .
\end{aligned}
$$

Here $x_{P}$ is the $\mathrm{OH}$ stretch coordinate of the ion at position $P$, i.e., it is equal to $\Delta r_{\mathrm{OH}}=r(\mathrm{OH})-r_{e}(\mathrm{OH})$ for that ion, and $w_{P, P^{\prime}}\left(x_{P}, x_{P^{\prime}}\right)$ is the interaction between ions $P$ and $P^{\prime}$. The quantity $\mu$ is the reduced mass of an $\mathrm{OH}$ group and $w_{P, P}\left(x_{P}, x_{P}\right)=0$. Intuitively one would expect that the stretching motions of the two $\mathrm{OH}$ bonds are coupled to the corresponding librational motions, but it may be argued that these librations mainly cause some shortening of the stretching 
potential $v\left(x_{P}\right)$ and some softening of the interactions $w_{P, P^{\prime}}\left(x_{P}, x_{P^{\prime}}\right)$.

For the remainder of this paper it is important to notice that the Hamiltonian governing the dynamics of the crystal is determined only up to an additive constant. The Hamiltonian in Eq. (1) may therefore be chosen to be the total crystalHamiltonian (with the usual definition of the zero of energy) minus the potential energy obtained with all degrees of freedom taken at their equilibrium values. Although calculation of the latter calls for the evaluation of Madelung sums to include all long range electrostatic interactions, the remaining part of the potential results from small displacements of the atoms from their equilibrium positions and may safely be assumed to contain only short range contributions. Only in the case of longitudinal lattice modes with very long wavelengths, i.e., near the gamma-point of the Brillouin-zone, will this approximation be somewhat doubtful.

In Ref. 8, the potential surface derived from density functional theory (DFT) calculations was presented as a function of the two $\mathrm{OH}$ stretch coordinates in each unit cell. Calculations were performed for an infinite crystal such that $x_{P}=x_{P^{\prime}}$ whenever $P$ and $P^{\prime}$ were on the same sublattice. The total potential energy per unit cell $V\left(x_{1}, x_{2}\right)$ obtained by these authors therefore reads in our notation,

$$
\begin{aligned}
V\left(x_{1}, x_{2}\right)= & v\left(x_{1}\right)+v\left(x_{2}\right)+3 w\left(x_{1}, x_{2}\right)+6 w^{\prime}\left(x_{1}, x_{1}\right) \\
& +3 w^{\prime \prime}\left(x_{1}, x_{2}\right)+\cdots
\end{aligned}
$$

The factor of 3 in front of the nearest neighbor interactions $w\left(x_{1}, x_{2}\right)$ appears because the two groups in each cell have one interaction among each other and in total four interactions with groups in neighboring cells, which each contribute half an interaction to the energy of one unit cell. Similar arguments lead to the factors with the next-nearest neighbors within one layer of $\mathrm{OH}$ groups $w^{\prime}\left(x_{1}, x_{1}\right)$ and the interactions across the Mg-layers $w^{\prime \prime}\left(x_{1}, x_{2}\right)$. Evidently we cannot calculate the single particle energy $v(x)$ and all the interaction energies individually without having further information or making further approximations. We therefore make the following educated approximations. First of all, from the fact that harmonic dispersion curves show little or no dispersion for wave vectors along the $\vec{c}$-axis (Fig. 4, upper part) we conclude that interactions between $\mathrm{OH}$ groups in different layers are very small. We will therefore assume that $w^{\prime \prime}\left(x_{1}, x_{2}\right)=0$, and consequently that the crystal consists of independent layers of $\mathrm{OH}$ groups stacked along the $\vec{c}$-axis. We thus find it useful to envisage the collection of $\mathrm{OH}$ groups, or oscillating particles, as forming a collection of layers stacked along the crystal $\vec{c}$-axis. Nearest neighbors in such a layer are connected to different $\mathrm{Mg}^{2+}$ layers, as shown in Fig. 1. The Hamiltonian given above may then be restricted to one such layer of antiparallel $\mathrm{OH}$ groups, implying that $P=(\vec{n}, i)$ with $\vec{n}$ indicating a two-dimensional (2D) lattice cell and $i$ a sublattice. One way of determining the remaining terms would be as follows. First, choose the stretch potential of an individual $\mathrm{OH}$ group in the gas phase as a reference potential. Next attach the ion to a Mg-layer as it appears in the brucite crystal. This will slightly change the reference potential by an amount $\delta v(x)$, which depends only

\section{2-D PES for $\mathrm{OH}$ vibrational coupling in the $\mathrm{Mg}(\mathrm{OH})_{2}$ crystal from DFT calculations}

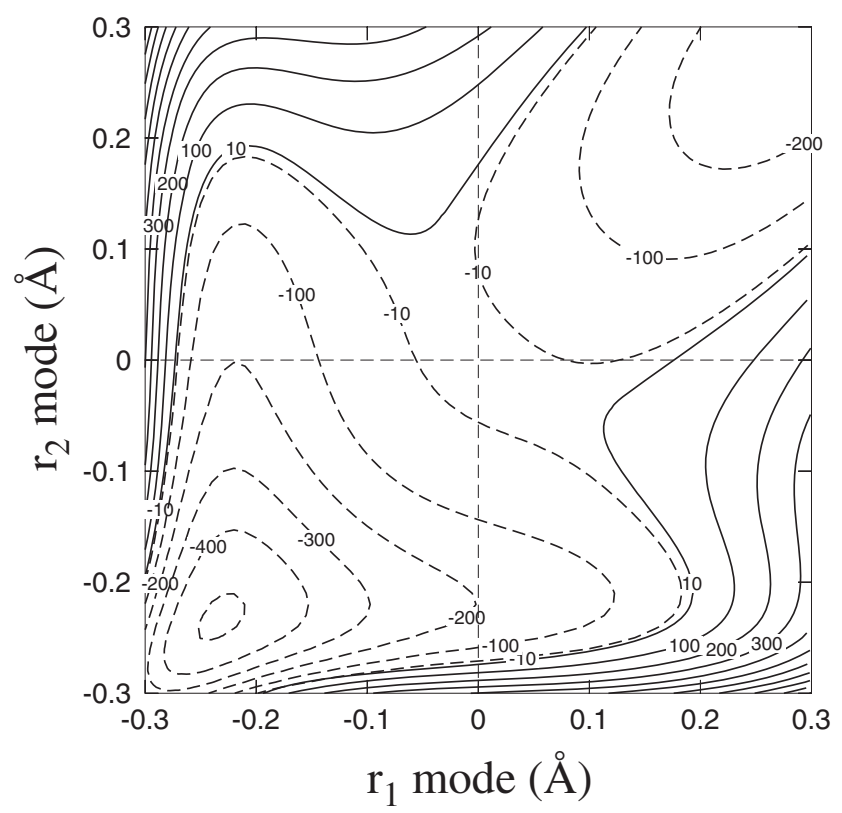

FIG. 3. 2D potential energy surface of the function $V\left(x_{1}, x_{2}\right)-v\left(x_{1}\right)-v\left(x_{2}\right)$, where $V\left(x_{1}, x_{2}\right)$ is shown in Fig. 2 and $v\left(x_{1}\right)$ and $v\left(x_{2}\right)$ are Morse functions as defined in Sec. II A.

on the stretch coordinate $x$; besides this it will introduce next-nearest neighbor interactions depending on two coordinates. Finally, combine a collection of Mg-layers with attached $\mathrm{OH}$ groups to construct the brucite crystal. This will introduce the nearest neighbor interactions mentioned above and possibly slightly change the single particle energy again. From a model describing the interactions, for example a model based on mutual polarization of the $\mathrm{OH}$ groups, one may infer the relative importance of the nearest and nextnearest neighbor contributions and finally calculate them individually. Notice that the stretch coordinates describe the difference of the various $\mathrm{OH}$ distances with respect to their equilibrium value, implying that all interaction terms are at least of the $\delta \vec{d}_{P}-\delta \vec{d}_{P^{\prime}}$ type, with $\delta \vec{d}_{P}=\vec{d}_{P}\left(x_{P}\right)-\vec{d}_{P}(0)$, and $\vec{d}_{P}\left(x_{P}\right)$ being the dipole at point $P$. Consequently the interactions will quickly become smaller with increasing distance between the ions. We therefore assume that we can restrict our Hamiltonian to include only nearest neighbor interactions, so $w_{P, P^{\prime}}\left(x_{P}, x_{P^{\prime}}\right)=w\left(x_{P}, x_{P^{\prime}}\right)$ for nearest neighbors and zero otherwise.

Still, in $V\left(x_{1}, x_{2}\right)=v\left(x_{1}\right)+v\left(x_{2}\right)+3 w\left(x_{1}, x_{2}\right)$, we must discriminate between $v(x)$ and $w\left(x_{1}, x_{2}\right)$. To this end we simply assume that the on-site potential $v(x)$ is by definition given by a Morse potential function,

$$
v(x)=D\left(1-e^{-a x}\right)^{2} .
$$

The parameters $D$ and $a$ are chosen such that $V\left(x_{1}, x_{2}\right)$ is best approximated by a sum of two of these functions. The remaining part $V\left(x_{1}, x_{2}\right)-v\left(x_{1}\right)-v\left(x_{2}\right)$ is then by definition equal to $3 w\left(x_{1}, x_{2}\right)$. A plot of the interaction term $w\left(x_{1}, x_{2}\right)$ is shown in Fig. 3. By comparing Figs. 2 and 3 one realizes that the interaction is actually very small and that splitting of 
degenerate vibration states due to interactions will be very small. Notice that for $x=0$, i.e., when $r(\mathrm{OH})=r_{e}(\mathrm{OH})$, the Morse potential is equal to zero. With our choice of the zero of potential energy discussed above, this implies that $w(0,0)=0$ as well. Moreover, notice that the Morse potential remains finite when $x=-r_{e}(\mathrm{OH})$, i.e., when $r(\mathrm{OH})=0$. This is a well known artifact of the Morse potential, which has no effect on the description of bound states since $D$ is very large.

The consequences of the approximations introduced to get access to the complete crystal Hamiltonian, complete as far as the $\mathrm{OH}$ stretchings are concerned, are modest. First, since the total potential energy per unit cell in our model is the same as in the original model, all calculations at the $\Gamma$-point will produce the same results as before. Second, attributing the complete interaction energy to the first nearest neighbors will result in dispersion curves which are smoothed representations of the real dispersion curves. We leave a calculation of the, presumably small (see previous paragraph), additional structure of the dispersion curves for future work.

It is perhaps worth mentioning that the procedure, outlined below, to calculate the dispersion curves in no way is limited to the present Hamiltonian. It can easily be applied to a more complex three-dimensional (3D) Hamiltonian with more complicated interactions.

\section{B. Crystal excitations}

We now continue with a description of the calculation of low-lying excitation energies of the Hamiltonian just defined. In principle our task is to choose a basis for the crystal as a whole and to diagonalize the Hamiltonian in this basis. In order to define a crystal basis, we first choose an on-site basis, i.e., a set of basis functions, at each point $P$, and next construct crystal functions by taking products of such functions, one factor for each point $P$. As already stated above, in order to be allowed to use the Brillouin theorem, we should use single particle mean-field states to construct our basis of crystal functions. We have found however that the mean-field functions hardly differ from the Morse oscillator functions corresponding to the Morse potential introduced above. We therefore make use of the latter throughout this subsection, understanding that the more correct expressions may be obtained by everywhere replacing Morse oscillator functions by mean-field functions. The numerical results obtained using these more exact equations will be given in the various tables as well. It will be seen that the results so obtained hardly differ from those obtained with the Morse oscillator functions.

As a first step in diagonalizing the full Hamiltonian, we construct crystal basis functions adapted to the translational symmetry of the crystal. Restricting ourselves to the crystal ground state and first excited states we have

$$
\begin{aligned}
& |\Phi\rangle=\prod_{P}|0\rangle_{P}, \\
& \left|\Phi_{\vec{q}}^{i}\right\rangle=\frac{1}{\sqrt{N}} \sum_{\vec{n}} e^{i \vec{q} \cdot \vec{R}_{\vec{n}}} E_{(\vec{n}, i)}^{*}|\Phi\rangle .
\end{aligned}
$$

Here $E_{P}^{*}$ is an excitation operator, replacing $|0\rangle_{P}$ by $|1\rangle_{P}$ in the product just following it; $\vec{q}$ is a wave vector from the first Brillouin zone and $\vec{R}_{\vec{n}}$ the position vector of the unit cell indexed by $\vec{n} . N=M_{x} M_{y}$ is the number of unit cells in the $2 \mathrm{D}$ crystal. The functions $|i\rangle$ are the Morse oscillator functions of which the first three are given in Table I, together with the corresponding energies.

Because the matrix elements between $|\Phi\rangle$ and the singly excited states are (approximately) zero, the ground state will simply be $|\Phi\rangle$ with corresponding energy

$$
E_{0}=2 N \varepsilon_{0}+3 N\langle 00|w| 00\rangle .
$$

The matrix element $\langle 00|w| 00\rangle$ is just $w\left(x_{1}, x_{2}\right)$ sandwiched between products of two Morse oscillator ground states, once in the bra and once in the ket, and integrated over the two variables $x_{1}$ and $x_{2}$. A similar notation will be used below for similar integrals, for example $\langle 01|w| 10\rangle$ represents the integral of $w\left(x_{1}, x_{2}\right)$ sandwiched between a bra consisting of the product of a Morse oscillator ground state and a Morse oscillator excited state, and a ket consisting of the product of the same two functions in reverse order.

Since the crystal basis functions have been adapted to the translational symmetry of the lattice, the Hamiltonian is block diagonal, one block for each $\vec{q}$ vector. Choosing the unit cell such that the two $\mathrm{OH}$ groups are positioned at $\vec{r}_{1}$ $=\vec{a} / 3+2 \vec{b} / 3+z_{1} \vec{c}$ and $\vec{r}_{2}=2 \vec{a} / 3+\vec{b} / 3+z_{2} \vec{c}$, respectively, with $\vec{a}, \vec{b}$, and $\vec{c}$ being unit cell vectors, the reciprocal lattice vectors are $\vec{a}^{*}=(4 \vec{a}+2 \vec{b}) /\left(3 a^{2}\right), \vec{b}^{*}=(2 \vec{a}+4 \vec{b}) /\left(3 a^{2}\right)$, and $\vec{c}^{*}$ $=\vec{c} / c^{2}$. The $\vec{q}$ block of $H-E_{0}$ then reads

$\left(H-E_{0}\right)_{\vec{q}}=\left[\begin{array}{cc}\varepsilon_{1}-\varepsilon_{0}+3\langle 01|w| 01\rangle-3\langle 00|w| 00\rangle & \left(1+e^{-i \vec{q} \cdot \vec{a}}+e^{i \vec{q} \cdot \vec{b}}\right)\langle 01|w| 10\rangle \\ \left(1+e^{i \vec{q} \cdot \vec{a}}+e^{-i \vec{q} \cdot \vec{b}}\right)\langle 01|w| 10\rangle & \varepsilon_{1}-\varepsilon_{0}+3\langle 01|w| 01\rangle-3\langle 00|w| 00\rangle\end{array}\right]$.

On diagonalizing this matrix we find the excitation energies

$$
\begin{aligned}
& \hbar \omega(\vec{q})_{g}=\varepsilon_{1}-\varepsilon_{0}+3\langle 01|w| 01\rangle-3\langle 00|w| 00\rangle+\langle 01|w| 10\rangle \sqrt{3+2 \cos (\vec{q} \cdot \vec{a})+2 \cos (\vec{q} \cdot \vec{b})+2 \cos (\vec{q} \cdot(\vec{a}+\vec{b}))}, \\
& \hbar \omega(\vec{q})_{u}=\varepsilon_{1}-\varepsilon_{0}+3\langle 01|w| 01\rangle-3\langle 00|w| 00\rangle-\langle 01|w| 10\rangle \sqrt{3+2 \cos (\vec{q} \cdot \vec{a})+2 \cos (\vec{q} \cdot \vec{b})+2 \cos (\vec{q} \cdot(\vec{a}+\vec{b}))} .
\end{aligned}
$$


Letting $\vec{q}=q \vec{a}^{*}$ we have

$$
\begin{aligned}
\hbar \omega\left(q \vec{a}^{*}\right)_{g}= & \varepsilon_{1}-\varepsilon_{0}+3\langle 01|w| 01\rangle-3\langle 00|w| 00\rangle \\
& +\langle 01|w| 10\rangle \sqrt{5+4 \cos (q)}, \\
\hbar \omega\left(q \vec{a}^{*}\right)_{u}= & \varepsilon_{1}-\varepsilon_{0}+3\langle 01|w| 01\rangle-3\langle 00|w| 00\rangle \\
& -\langle 01|w| 10\rangle \sqrt{5+4 \cos (q)} .
\end{aligned}
$$

Other examples can easily be calculated.

We end this section discussing possible corrections to the ground state from doubly excited states. Within the approximations accepted so far, no correlations have been included in the crystal ground state between the $\mathrm{OH}$ groups in different cells. While studying phonons in $\alpha-N_{2}{ }^{12-14}$ it was found that with such a method acoustic phonons do not converge to zero frequency at the center of the Brillouin zone. Using the RPA method, which basically incorporates doubly excited states in the crystal ground state, could salvage this problem. This extension of the method, however, had only small consequences for the energies of the low lying optical modes and no noticeable consequences at all for the energies of the higher optical modes. Since in this paper we are concerned with optical modes of rather high energies, it is not useful to extend our calculations in this direction.

\section{Selection rules}

The infrared absorption intensity is proportional to the Fourier transform of the time autocorrelation function of the total dipole moment of the (2D) crystal:

$$
I(\omega) \propto \int d t e^{i \omega t}\langle\vec{M}(0) \cdot \vec{M}(t)\rangle .
$$

Here $\vec{M}$ is the total dipole moment of the crystal. Obviously, in our system the average dipole moment is zero; the infrared absorption intensity, however, is proportional to the fluctuations of the dipole moment and in general differs from zero. In Appendix B it will be shown that the time dependent autocorrelation function of the total dipole moment may be reduced to

$$
\langle\vec{M}(0) \vec{M}(t)\rangle=2 N \delta_{\vec{q}, 0} \delta_{\alpha, u}\langle 0|\vec{\mu}| 1\rangle \cdot\langle 1|\vec{\mu}| 0\rangle e^{-i \hbar \omega(\overrightarrow{0})_{u^{t}}} .
$$

Here $\vec{\mu}$ is the single particle dipole moment operator. We conclude from this result that IR absorption experiments measure the excitation to the ungerade state at the center of the Brillouin zone.

Similarly, the Raman scattering intensity is proportional to the Fourier transform of the time dependent autocorrelation of the total polarizability tensor sandwiched between the polarization vectors of the incoming and outgoing light beams. In our model we only have access to the $z z$-component $A_{z z}$ of the total polarizability tensor of the crystal. The scattered intensity then reads

$$
\frac{d \sigma}{d \omega} \propto \int d t e^{i \omega t}\left\langle A_{z z}(0) A_{z z}(t)\right\rangle .
$$

In Appendix B it is shown that the relevant time correlation function may be calculated as

$$
\left\langle A_{z z}(0) A_{z z}(t)\right\rangle=2 N \delta_{\vec{q}, \overrightarrow{0}} \delta_{\alpha, g}\left\langle 0\left|\alpha_{z z}\right| 1\right\rangle \cdot\left\langle 1\left|\alpha_{z z}\right| 0\right\rangle e^{-i \hbar \omega(\overrightarrow{0})_{g} t}
$$

Here $\alpha_{z z}$ is the zz-component of the single particle polarizability tensor. We conclude that Raman scattering experiments measure excitations to the first excited gerade vibrational state with $\vec{q}=\overrightarrow{0}$.

\section{HARMONIC CALCULATIONS}

In order to asses the validity of our claim that the ensemble of all $\mathrm{OH}$ groups in the crystal can be considered to form a collection of uncoupled 2D layers of $\mathrm{OH}$ groups stacked along the $\vec{c}$-axis, we have performed harmonic lattice dynamics calculations. For our claim to be true, it is necessary that there be little or no dispersion along the $\vec{c}$-axis. A second reason to perform these calculations is that the results provide us with a valuable reference with which we can compare our anharmonic frequency dispersion curves. Since for these purposes it is important to have access to complete dispersion curves and not just frequencies at isolated points, we chose to use a code which has these options, even though this meant that the potential energy surface on which the calculations are based is a bit different from the one used thrust of this paper (namely for the anharmonic calculations). We have checked that at isolated points the results with this code do not differ much from results obtained with the actual PES used in this paper.

We have performed our ab initio lattice dynamics (LD) calculations within the framework of a density functional perturbation theory (DFPT) approach, using plane waves and pseudopotentials and the PW91-GGA functional encoded in CASTEP. ${ }^{16}$ Details of the calculations are given in Ref. 17. The results for those modes that are dominated by the $\mathrm{OH}$ stretch coordinates are represented by the drawn line in the upper panel of Fig. 4. It is seen that in those parts of the plot where only the component of $\vec{q}$ along the $\vec{c}$-axis is changing only small variations occur in the plotted frequencies. This confirms to a large extent that the motions of the $\mathrm{OH}$ groups on opposite sides of the $\mathrm{Mg}$-layers are hardly coupled.

The dashed line in this same panel represents the results obtained with a harmonic model with all degrees of freedom fixed to their equilibrium values except for the $z$-coordinates of the two oxygen and the two hydrogen atoms. We will refer to this model as the $4 D$ model (four-dimensional model). It is seen that restricting the degrees of freedom has no influence on the Raman or gerade mode and, roughly speaking, shifts the infrared (IR) or ungerade mode upward by a small amount. This is intuitively clear since in the gerade mode possible displacements of the $\mathrm{OH}$ centers of mass are in the opposite direction and therefore their sum does not couple to the $\mathrm{Mg}$ displacements. In the IR mode, on the other hand, the sum of the two $\mathrm{OH}$ centers of mass is not restricted by symmetry to be zero, and therefore may couple to the motion of the $\mathrm{Mg}$ ion. The additional degree of freedom then leads to a softening in the latter case. Notice that as a result of this shift upward the energy difference between the gerade and ungerade modes has become the same in the two parts of the plot where only the component of $\vec{q}$ along $\vec{c}$ varies. Moreover the 
crossing of the two modes shifted a little bit toward the zone boundary. The LO-TO split of the gerade mode will be discussed in Sec. V B.

\section{POTENTIAL ENERGY SURFACE FOR THE ANHARMONIC CALCULATIONS}

The PES used in the present work is taken from Ref. 8 and we refer to that paper for the technical details concerning the underlying DFT calculations, which used a GGA type functional of the PW91 type and ultrasoft pseudopotentials, and were performed with the VASP package. ${ }^{18-20}$ Here only some of the basic information will be repeated. The crystal structure was optimized and our cell parameters are in good agreement with experiment and differ by about $1 \%$ from the experimental $15 \mathrm{~K}$ parameters of Chakoumakos et al. ${ }^{21}$ and the calculated cell volume is about $3 \%$ larger.

The potential energy grid, $V\left(x_{1}, x_{2}\right)$, was tabulated between -0.3152 and $0.3152 \AA$ in steps of $\Delta x=0.03152 \AA$. In the second step, a sum of two Morse potential functions, $v\left(x_{1}\right)+v\left(x_{2}\right)$, one for $\mathrm{OH} 1$ and one for $\mathrm{OH} 2$, were fitted to the PES energies (cf. Sec. II A). In the third step, the function $V\left(x_{1}, x_{2}\right)-v\left(x_{1}\right)-v\left(x_{2}\right)$ was calculated at each grid point, and a spline-type function consisting of smooth fourth order polynomials connecting the new grid point energies was calculated. By definition this was set equal to $3 w\left(x_{1}, x_{2}\right)$ as mentioned in Sec. II A.

\section{RESULTS AND DISCUSSION}

\section{A. Excitations at the $\Gamma$ point and basis-set convergence}

There are two symmetry-allowed normal modes involving the $\mathrm{OH}$ stretchings in the $P \overline{3} m 1$ crystal structure of brucite: the symmetric Raman-active $\mathrm{A}_{1 \mathrm{~g}}$ mode and the antisymmetric IR-active $\mathrm{A}_{2 \mathrm{u}}$ mode. The experimental (anharmonic) frequencies of the Raman mode is $\sim 3652 \mathrm{~cm}^{-1}$ (see, e.g., Ref. 22), and of the IR mode $\sim 3698 \mathrm{~cm}^{-1}$ (see, e.g., Ref. 23). These frequencies have been addressed from a theoretical point in Ref. 8. In this subsection we redo these calculations, but now using a Morse oscillator basis rather than a simple harmonic oscillator basis.

We have diagonalized the cell-Hamiltonian,

$$
H_{\text {cell }}\left(x_{1}, x_{2}\right)=h\left(x_{1}\right)+h\left(x_{2}\right)+3 w\left(x_{1}, x_{2}\right),
$$

in a basis of Morse oscillator functions of increasing size. The largest basis that we used was $\{|00\rangle,|10\rangle,|01\rangle,|11\rangle,|20\rangle,|02\rangle\}$. Because of the symmetry of the Hamiltonian it has been useful to split the basis in a symmetric part $\left\{\Sigma_{1}, \Sigma_{2}, \Sigma_{3}, \Sigma_{4}\right\}$ and an antisymmetric part $\left\{A_{1}, A_{2}\right\}$, with

$$
\begin{array}{ll}
\Sigma_{1}=|00\rangle, & \\
\Sigma_{2}=\{|10\rangle+|01\rangle\} / \sqrt{2}, & A_{1}=\{|10\rangle-|01\rangle\} / \sqrt{2}, \\
\Sigma_{3}=|11\rangle, & A_{2}=\{|20\rangle-|02\rangle\} / \sqrt{2},
\end{array}
$$

$$
\Sigma_{4}=\{|20\rangle+|02\rangle\} / \sqrt{2} .
$$

The results of the diagonalization are given in Table II, together with those of a diagonalization in $\left\{\Sigma_{1}, \Sigma_{2}, A_{1}\right\}$. It is seen that the ground state energy and excited state energies are pretty much the same with both methods, from which we conclude that the single particle basis functions can be restricted to the Morse oscillator ground state and first excited state. Notice that frequencies, i.e., excitation energies, converge even faster than the individual energies.

We also calculated the frequencies using increasingly large numbers of harmonic oscillator basis functions (and the program VIB2D). ${ }^{8}$ Not surprisingly, many more harmonic oscillator functions, in actual fact $8 \times 8$, i.e., 64 products of single particle basis function, are needed to reach convergence. There is an additional point that we want to mention. In order to get converged results in the harmonic oscillator basis, many excited harmonic oscillator functions are needed. Since these functions differ from zero at increasingly larger values of the coordinates, also $w\left(x_{1}, x_{2}\right)$ must be known with sufficient precision at increasingly larger values of the coordinates in order to be able to calculate matrix elements of the Hamiltonian correctly. The Morse oscillator functions are much more efficient in this respect.

It is interesting to have a look at the results in the even more restricted basis $\left\{\Sigma_{2}, A_{1}\right\}$, i.e., without allowing $\Sigma_{1}$ to mix with $\Sigma_{2}$. Also these energies turn out to be almost exact and the frequencies even better. From this fact we conclude that, even though the Brillouin theorem does not apply exactly in the Morse oscillator basis, it is completely safe to use it anyhow. Indeed the calculation in this very restricted basis is equivalent to that of Sec. II B, restricted to the $\Gamma$ point. The exact results in this case are

$$
\hbar \omega_{g}=\varepsilon_{1}-\varepsilon_{0}+3\langle 01|w| 01\rangle-3\langle 00|w| 00\rangle+3\langle 01|w| 10\rangle,
$$

$$
\hbar \omega_{u}=\varepsilon_{1}-\varepsilon_{0}+3\langle 01|w| 01\rangle-3\langle 00|w| 00\rangle-3\langle 01|w| 10\rangle .
$$

It must be pointed out that these formulas are extremely simple, which pinpoints the merits of the approach we present in this paper.

Incidentally, the absolute values of the frequencies differ from experiment by about a hundred $\mathrm{cm}^{-1}$. These discrepancies with respect to experiments must be due to inaccuracies of the electronic calculations or to coupling of the investigated modes with some or all other crystal vibrations.

\section{B. Dispersion curves}

In the previous subsection we have found that the basis per unit cell may be restricted to $\left\{\Sigma_{1}, \Sigma_{2}, A_{1}\right\}$ and that the Brillouin theorem may be applied. The latter has been substantiated by results obtained with the mean-field method described in Appendix A. We therefore continue to calculate dispersion curves using the theory outlined in Sec. II.

Using Eqs. (8a) and (8b) we have calculated anharmonic phonon dispersion curves for the $\mathrm{A}_{1 \mathrm{~g}}$ and $\mathrm{A}_{2 \mathrm{u}} \mathrm{OH}$ modes along the main directions in the Brillouin zone. The results 

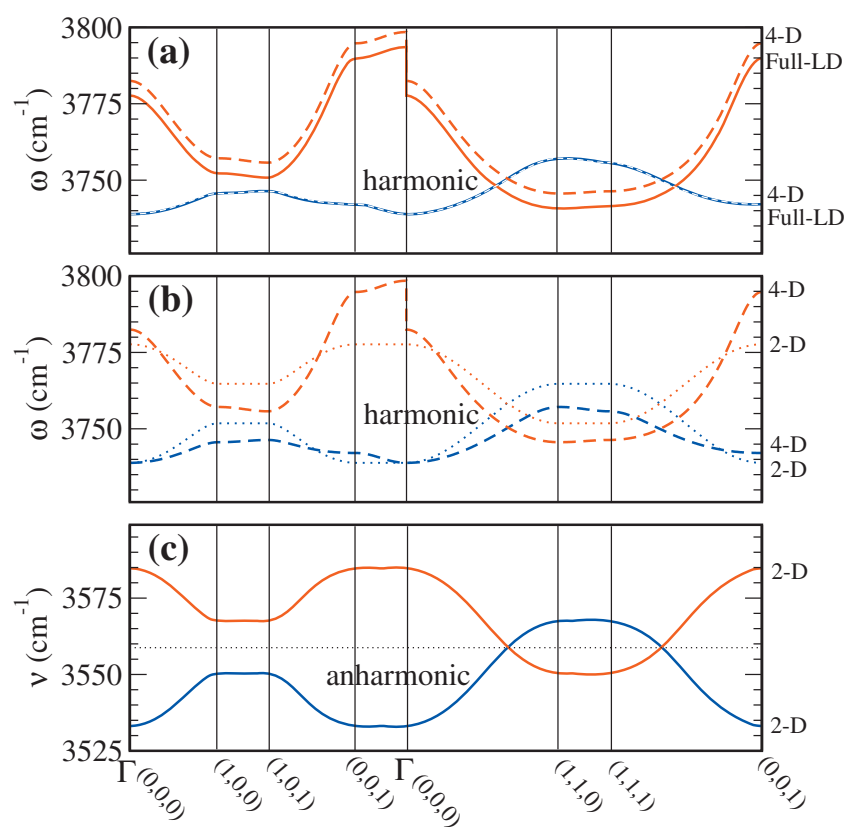

FIG. 4. Comparison of our resulting anharmonic dispersion curves [in (c)] with various harmonic models of different sophistication [in (a) and (b)], as explained in the text. The anharmonic curves were calculated from Eqs. (8a) and (8b), based on the DFT-generated 2D potential energy surfaces shown in Figs. 2 and 3. The harmonic dispersion curves were calculated from lattice dynamics calculations including either all vibrational degrees of freedom of the whole crystallographic unit cell ("full-LD"), or only four ("4D") or two (“2D") degrees-of-freedom (see text for details).

are presented in the lower part of the lower panel of Fig. 4. Since, to the best of our knowledge, there are no experimentally measured dispersion curves of the $\mathrm{OH}$ stretching frequencies in brucite, we will compare our results with those of harmonic calculations. For this purpose we have presented in the upper part of this same panel the results obtained with the restricted, 4D harmonic model presented in Sec. III, and those of yet another, even more restricted, 2D harmonic model in which only the two $\mathrm{OH}$ stretching degrees of freedom are taken into account and next-nearest neighbor interactions are ignored.

One distinctive difference in the features of the various curves is the characteristic split for ionic materials between the longitudinal optical (LO) and transverse optical (TO) modes at the $\Gamma$ point occurring in the $4 \mathrm{D}$ harmonic model but not in the other two models. This type of splitting occurs when charged groups on different sublattices move with re- spect to each other at very long wavelengths. It is a result of long range interactions producing macroscopic fields that couple much stronger to the longitudinal modes than to the transversal modes. In the harmonic calculations, in the long wavelength limit, the coupling between the phonons and the macroscopic electric fields is reproduced by including corrections to the dynamical matrix. ${ }^{24,25}$ The basic assumption made in both the 2D harmonic model and in the new anharmonic model is that the stretching dynamics of all $\mathrm{OH}$ ions in the crystal can be studied independently from the dynamics of all other degrees of freedom. As we have argued in Sec. II A, this implies that no long range interactions occur in the corresponding Hamiltonians and hence that no LO-TO splitting is observed. However, even if the presumed weak coupling between the $\mathrm{OH}$ stretches and the remaining degrees of freedom may be expected to have little or no effects on the dynamics of the stretch coordinates at most wavelengths this may not be true at very large wavelengths. In that case, even a tiny coupling to the motions of other charged objects will lead to LO-TO splitting. An example of such coupling is the correlated dynamics of the $\mathrm{OH}$ stretching coordinates and the displacements of their centers of mass. The charged $\mathrm{OH}$ ions will then move with respect to each other and with respect to the charged Mg ions.

Another difference between our results and those of the harmonic calculations is the rather strong symmetry in our dispersion curves. They display perfect mirror symmetry with respect to the dashed line shown in the figure. This is a consequence of the fact that in the end our theoretical treatment leads to the diagonalization of a two-dimensional matrix for every $\vec{q}$-vector. The same holds true for the harmonic calculations if they are restricted to include only stretching degrees of freedom, i.e., for the 2D harmonic model. In our anharmonic model the dimension of the matrices that we diagonalize is set by the number of excitations used per unit cell. As we have seen, in the present case it is sufficient to excite each of the two independent $\mathrm{OH}$ groups to their first excited states, so we need two states per unit cell. Had it been necessary to include excitations to the second excited Morse oscillator state, we would have been left with a four dimensional matrix for every $\vec{q}$-vector. The mirror symmetry observed in the present calculations would then have been broken. Similarly, of course if we had included additional degrees of freedom, like for example the centers of mass

TABLE II. Comparison of the resulting energy levels using different bases.Two decimals are given only to underline the small differences in our results. The results from a first order perturbation calculation are seen to be identical to the results with a small basis.

\begin{tabular}{|c|c|c|c|c|c|}
\hline \multicolumn{2}{|c|}{ Small basis } & \multicolumn{2}{|c|}{ Large basis } & \multicolumn{2}{|c|}{ Mean field } \\
\hline$E_{i}$ & Freq. & $E_{i}$ & Freq. & $E_{i}$ & Freq. \\
\hline 3712.58 & & 3712.48 & & 3712.58 & \\
\hline 7245.72 & 3533.14 & 7245.33 & 3532.85 & 7245.72 & 3533.14 \\
\hline \multirow[t]{4}{*}{7297.31} & 3584.74 & 7297.13 & 3584.65 & 7297.31 & 3584.73 \\
\hline & & 10628.02 & & & \\
\hline & & 10643.12 & & & \\
\hline & & 10849.62 & & & \\
\hline
\end{tabular}


coordinates of all $\mathrm{OH}$ groups. In the latter case also the harmonic calculations loose their mirror symmetry as seen from the results with the $4 \mathrm{D}$ harmonic model.

It is important to realize that including interactions other than nearest neighbor interactions within the $\mathrm{OH}$ layers will not lift the mirror symmetry, neither in our approach, nor in the harmonic calculations. It may on the other hand introduce more structure in the dispersion curves, which are now rather "sinusoidal." Apart from the LO-TO splitting the dispersion curves of the 4D harmonic model resemble those of the other two models rather much. In particular the 4D model has its gerade and ungerade curves cross at the same point as the other two models, even though it includes next-nearest neighbor interactions while the others do not. From this we conclude that the interactions between next nearest neighbors within the $\mathrm{OH}$ layers are negligible. On the other hand including the $\mathrm{OH}$ centers of mass coordinates in the 4D harmonic model allows for some softening in both modes. This appears to occur mainly on approaching the Brillouin zone boundaries.

One more point to mention is that if one just plots the results from Eqs. (8a) and (8b) one will observe an apparent avoided crossing at every point where the argument of the square root becomes zero. A quick glance at the corresponding eigenvectors will prove that they switch symmetry as well and that the curves actually do cross.

\section{Isotope isolated systems}

In order to probe local environments in crystals, experimentalists often study the dynamics of an $\mathrm{OH}$ group in an otherwise deuterated crystal. Assuming that the $\mathrm{OH}$ stretch is uncoupled from the dynamics of the crystal and only probes the potential energy provided by the surrounding OD-groups, the first excitation energy may easily be shown to be

$$
\hbar \omega_{\text {iso }}=\varepsilon_{1}-\varepsilon_{0}+3\left\langle 0_{D} 1_{H}|w| 0_{D} 1_{H}\right\rangle-3\left\langle 0_{D} 0_{H}|w| 0_{D} 0_{H}\right\rangle .
$$

In our calculations again we have restricted ourselves to using the Morse oscillator ground and excited states. The subscripts at the Morse oscillator indices indicate the type of oscillator they refer to, i.e., either OD or OH. Since the matrix elements of $w(x, y)$ hardly depend on the reduced masses on the one hand, and since the single-particle mean-field functions hardly differ from the Morse oscillator functions on the other hand, the isotope-isolated frequency $\omega_{\text {iso }}$ is expected to be approximately equal to $\left(\omega_{g}+\omega_{u}\right) / 2$.

\section{SUMMARY AND CONCLUDING REMARKS}

In many cases when mode-following is not a good approach, it may be possible to select not just one, but two or more coordinates, not necessarily phonon coordinates, such that the full Hamiltonian separates in two independent terms, one depending on the selected coordinates and the other independent of these. In such cases the selected coordinates may be studied independently from all other coordinates. This is the approach that we adopted in the present paper. We assumed that the collection of all $\mathrm{OH}$ stretch coordinates constitutes a set of $2 M$ coordinates, with $M$ being the num- ber of unit cells in the crystal, well separated from all remaining coordinates. Using these coordinates we have proposed a Hamiltonian that we treated in as exact manner as possible. We have assumed that each infinite layer consisting of two antiparallel $\mathrm{OH}$ sublattices [Fig. 1(a)] are uncoupled from the others, so we were finally left with a 2D crystal Hamiltonian. Justification for this assumption comes from the fact that harmonic phonon calculations show little dispersion for $\vec{q}$-vectors along the crystal $\vec{c}$-axis.

The method that we have used to diagonalize the Hamiltonian contains two ingredients. First we have chosen a "quantum chemical" approach rather than an approach based on coordinate transformations as in the usual harmonic calculations. By quantum chemical approach we mean that we have chosen a set of crystal basis functions and diagonalized the Hamiltonian in this basis. The basis that we have chosen consist of one particular product of single particle functions, one factor for every degree of freedom, or particle, in the Hamiltonian, together with a set of "singly excited functions," one for each particle in the crystal. The singly excited function for particle $P$ is obtained from the original function by changing the factor at point $P$. The best set of single particle functions, from which to choose the factors in the crystal functions are the so-called mean-field functions. We have shown however that these mean-field functions hardly differ from a set of well chosen Morse oscillator functions. The second ingredient in our model is that we restrict it to include only nearest neighbor interactions. These two assumptions together allow us to perform the diagonalization analytically, apart from the evaluation of a few integrals. It is worth mentioning that the method is not restricted to the Hamiltonian studied in this paper. It may easily be used in 3D models including interactions beyond the nearest neighbors. In the case of nearest and next nearest neighbor interactions the diagonalization still can be done analytically without any problems.

At the $\Gamma$-point our results reduce to those of a previous paper, now obtained in a much simpler way and apt to a simple intuitive interpretation. One of the conclusions of the previous paper was that one-dimensional (1D) mode following in the present case is not a reliable method. It is therefore a bit surprising that recently such a calculation has been claimed to give good results for the isotope isolated case. In the present setting, i.e., ignoring correlations between $\mathrm{OH}$ stretches and other degrees of freedom, the suggested approach is to introduce new coordinates according to $\left\{q_{1}\right.$ $=\left(x_{1}+x_{2}\right) / 2$, $\left.q_{2}=\left(x_{1}-x_{2}\right) / 2\right\}$ and to investigate the motion along $q_{1}$, while keeping $q_{2}$ equal to zero. The correct cell-Hamiltonian in $q$-coordinates reads

$$
\begin{aligned}
H_{\text {cell }}\left(q_{1}, q_{2}\right)= & -\frac{\hbar^{2}}{4 \mu} \frac{\partial^{2}}{\partial q_{1}^{2}}+v\left(q_{1}+q_{2}\right)-\frac{\hbar^{2}}{4 \mu} \frac{\partial^{2}}{\partial q_{2}^{2}}+v\left(q_{1}-q_{2}\right) \\
& +3 w\left(q_{1}+q_{2}, q_{1}-q_{2}\right) .
\end{aligned}
$$

Taking $q_{2}=0$ we have 


$$
H_{\text {cell }}\left(q_{1}, 0\right)=-\frac{\hbar^{2}}{4 \mu} \frac{\partial^{2}}{\partial q_{1}^{2}}+2 v\left(q_{1}\right)+3 w\left(q_{1}, q_{1}\right) .
$$

Now, replacing both $\mu$ and $2 v\left(q_{1}\right)+3 w\left(q_{1}, q_{1}\right)$ by half their values, as was done in the cited paper, we arrive at the Hamiltonian of one single Morse oscillator with three halves of a small correction. This explains why the suggested method has produced seemingly reasonable results.

We have presented dispersion curves, both for our anharmonic model and for a series of harmonic models. In summary we have found that the anharmonic model, restricted to the $\mathrm{OH}$ stretching degrees of freedom has dispersion curves rather similar to those of the corresponding 2D harmonic model, with the latter shifted upward substantially and having slightly smaller splitting between the gerade and ungerade modes. Including couplings to the centers of mass motions of the $\mathrm{OH}$ groups allows for softening of both modes at the Brillouin zone boundaries and introduces rather strong LO-TO splitting near the center of the Brillouin zone. Finally adding all other degrees of freedom allows for softening of the ungerade or IR mode. The combination of the last two effects give the dispersion curves their rather asymmetric appearances.

From a technical point of view our model has a few important advantages. First, since our single particle functions are adjusted to the anharmonicity of the potential only very few single particle basis functions are needed. As a consequence all calculations have become very simple. Moreover, the potential energy needs to be known accurately only in the range of the Morse oscillator functions actually being used. Had these functions been represented by sums of harmonic oscillator functions many of the latter would have been needed, especially those having nonzero values near the periphery of the Morse functions. These would automatically have nonzero values even beyond the range of the Morse functions and would need accurate potential energy values also in these regions. Second, it turns out that the whole procedure is rather robust against variations in the single particle Morse potentials and the interaction potentials. For example, instead of the optimized Morse potential described in Sec. II, we used the gas-phase Morse potential also in the crystal, incorporating the difference in the perturbation, and found no difference in the final results.

In conclusion we think that despite the limitation in the approximation of the Hamiltonian, the results presented in this work, namely, the results for the anharmonic phonons dispersion, might be in particular interest for potential studies of heat transfer properties in hydrogen containing insulators.

\section{ACKNOWLEDGMENTS}

We would like to acknowledge the Swedish Research Council (VR) for financial support, the Swedish Infrastructure for Computing (SNIC) and the Uppsala Multidisciplinary Center for Advanced Computational Science (UPPMAX) for computing resources, and Uppsala University ("Centre for dynamical processes and structure formation") for traveling support.

\section{APPENDIX A: MEAN-FIELD CALCULATIONS}

The single-particle mean-field states may be expanded in any basis, preferably adapted as much as possible to the problem at hand. The best-suited basis for this purpose evidently is the one consisting of Morse wave functions, of which the first three are given in Table I. In Sec. V A we have shown that it is sufficiently accurate to restrict the calculations to linear combinations of the Morse oscillator ground and first excited state. The single-particle mean-field states then read

$$
\begin{gathered}
|\widetilde{0}\rangle=C_{00}|0\rangle+C_{01}|1\rangle, \\
|\widetilde{1}\rangle=C_{10}|0\rangle+C_{11}|1\rangle .
\end{gathered}
$$

The coefficients $\left\{C_{00}, C_{01}, C_{10}, C_{11}\right\}$ must be chosen such that

$$
\begin{aligned}
& h^{\mathrm{MF}}\left(x_{P}\right)|\tilde{i}\rangle=\varepsilon_{i}^{\mathrm{MF}}|\tilde{i}\rangle, \\
& h^{\mathrm{MF}}\left(x_{P}\right)=h\left(x_{P}\right)+\sum_{P^{\prime} \neq P}\left\langle\widetilde{0}\left|w\left(x_{P}, x_{P^{\prime}}\right)\right| \widetilde{0}\right\rangle_{P^{\prime}} .
\end{aligned}
$$

The bra and ket in $\left\langle\widetilde{0}\left|w\left(x_{P}, x_{P^{\prime}}\right)\right| \widetilde{0}\right\rangle_{P^{\prime}}$ refer to particle $P^{\prime}$. Evidently these equations must be solved iteratively, like any mean-field problem. We have performed these calculations and found only negligibly small differences between the mean-field states and the original Morse oscillator states. We therefore conclude that it is safe to use Morse oscillator functions throughout. We have performed the full calculations with mean-field functions as well and found no differences with respect to the ones presented in this paper.

\section{APPENDIX B: DETAILS OF THE CALCULATION OF SELECTION RULES}

The total dipole moment of the crystal is the sum of single particle dipole moments

$$
\vec{M}=\sum_{\vec{n}, i}(-1)^{i} \vec{\mu}_{\vec{n}, i} .
$$

The minus sign must be introduced because $\vec{\mu}_{\vec{n}, i}$ is a property of the $\mathrm{OH}$ group at site $P=\{\vec{n}, i\}$ whose direction depends on the sublattice number $i$. Stated differently, both dipoles in the unit cell point in opposite directions. The total dipole moment autocorrelation function then reads

$$
\begin{aligned}
\langle\vec{M}(0) \cdot \vec{M}(t)\rangle= & \sum_{\vec{n}, i} \sum_{\vec{n}^{\prime}, i^{\prime}}\left\langle\Phi\left|\vec{\mu}_{\vec{n}, i} \cdot e^{i\left(H-E_{0}\right) t} \vec{\mu}_{\vec{n}^{\prime}, i^{\prime}} e^{-i\left(H-E_{0}\right) t}\right| \Phi\right\rangle \\
& \times(-1)^{i+i^{\prime}} .
\end{aligned}
$$

We have subtracted the ground state energy from the Hamiltonian for later convenience; obviously it does not influence the final result. Within our approximation the (mean-field) ground state $|\Phi\rangle$ is the exact ground state with energy $E_{0}$. Therefore the rightmost propagator may be set equal to unity. We next introduce the resolution of the identity just before and just after the remaining propagator. Within the present model, 


$$
1=|\Phi\rangle\left\langle\Phi\left|+\sum_{\vec{q}, \alpha}\right| \Phi_{\vec{q}, \alpha}\right\rangle\left\langle\Phi_{\vec{q}, \alpha}\right|,
$$

where $\left|\Phi_{\vec{q}, \alpha}\right\rangle=\sum_{j}\left|\Phi_{\vec{q}}^{j}\right\rangle X_{j, \alpha}(\vec{q})$ is the exact excited state with wave vector $\vec{q}$ and of type $\alpha$, i.e., gerade or ungerade. Introducing the resolution of the identity as just announced, we find

$$
\begin{aligned}
\langle\vec{M}(0) \vec{M}(t)\rangle= & N^{2}\langle 0|\vec{\mu}| 0\rangle \cdot\langle 0|\vec{\mu}| 0\rangle \sum_{i, i^{\prime}}(-1)^{i+i^{\prime}} \\
& +\sum_{\vec{n}, i} \sum_{\vec{n}^{\prime}, i^{\prime}} \sum_{\vec{q}, \alpha} \sum_{j, j^{\prime}}\left\langle\Phi\left|\vec{\mu}_{\vec{n}, i}\right| \Phi_{\vec{q}}^{j}\right\rangle \\
& \cdot\left\langle\Phi_{\vec{q} j^{\prime}}^{j}\left|\vec{\mu}_{\vec{n}^{\prime}, i^{\prime}}\right| \Phi\right\rangle X_{j, \alpha}(\vec{q}) X_{j^{\prime}, \alpha}(\vec{q})^{*} e^{-i \hbar \omega(\vec{q})_{\alpha} t} \\
& \times(-1)^{i+i^{\prime}} .
\end{aligned}
$$

The asterisk indicates a complex conjugate. The first term is equal to zero because of the sums over the sublattices. In the second sum we introduce the definition of $\left|\Phi_{\vec{q}}^{j}\right\rangle$, obtaining

$$
\begin{aligned}
\langle\vec{M}(0) \vec{M}(t)\rangle= & \sum_{\vec{n}, i} \sum_{\vec{n}^{\prime}, i^{\prime}} \sum_{\vec{q}, \alpha} \frac{1}{N} e^{i \vec{q} \cdot\left(\vec{R}_{\vec{n}}-\vec{R}_{\vec{n}^{\prime}}{ }^{\prime}\right.}\langle 0|\vec{\mu}| 1\rangle \\
& \cdot\langle 1|\vec{\mu}| 0\rangle X_{i, \alpha}(\vec{q}) X_{i^{\prime}, \alpha}(\vec{q})^{*} e^{-i \hbar \omega(\vec{q}) \alpha^{t}(-1)^{i+i^{\prime}} .}
\end{aligned}
$$

Performing the sums over the lattice vectors $\vec{n}$ and $\vec{n}^{\prime}$ we get

$$
\begin{aligned}
\langle\vec{M}(0) \vec{M}(t)\rangle= & N \delta_{\vec{q}, \overrightarrow{0}}\langle 0|\vec{\mu}| 1\rangle \cdot\langle 1|\vec{\mu}| 0\rangle \sum_{\alpha} \sum_{i, i^{\prime}}(-1)^{i+i^{\prime}} X_{i, \alpha}(\overrightarrow{0}) \\
& \times X_{i^{\prime}, \alpha}(\overrightarrow{0})^{*} e^{-i \hbar \omega(\overrightarrow{0})_{\alpha} t} .
\end{aligned}
$$

Finally, the eigenvectors for the exact states are particularly simple for $\vec{q}=\overrightarrow{0}$ :

$$
X_{1, g}(\overrightarrow{0})=\frac{1}{\sqrt{2}}, X_{2, g}(\overrightarrow{0})=\frac{1}{\sqrt{2}}, X_{1, u}(\overrightarrow{0})=\frac{1}{\sqrt{2}}, X_{2, u}(\overrightarrow{0})=-\frac{1}{\sqrt{2}} .
$$

Using these in our last expression for the dipole moment autocorrelation function we get the final result stated in the main text.

The total polarizability again is the sum of single particle polarizabilities

$$
A_{z z}=\sum_{\vec{n}, i} \alpha_{z z ; \vec{n}, i} .
$$

This time no minus sign corresponding to the sublattice index should be used, since the induced dipoles on both sublattices point in the same direction. The procedure followed above for the calculation of the dipole-dipole autocorrelation function may now be repeated literally. Ignoring a fully elastic contribution we obtain the result stated in the main text.

${ }^{1}$ B. Civalleri, P. Ugliengo, C. M. Zicovich-Wilson, and R. Dovesi, Z. Kristallogr. 224, 241 (2009).

${ }^{2}$ B. Winkler, V. Milman, and M. C. Payne, Miner. Mag. 59, 589 (1995).

${ }^{3} \mathrm{Ph}$. Baranek, A. Lichanot, R. Orlando, and R. Dovesi, Chem. Phys. Lett. 340, 362 (2001).

${ }^{4}$ F. Pascale S. Tosoni, C. Zicovich-Wilson, P. Ugliengo, R. Orlando, and R. Dovesi, Chem. Phys. Lett. 396, 308 (2004).

${ }^{5}$ P. Ugliengo, F. Pascale, M. Mérawa, P. Labéguerie, S. Tosoni, and R. Dovesi, J. Phys. Chem. B 108, 13632 (2004).

${ }^{6}$ K. Hermansson, G. Gajewski, and P. D. Mitev, J. Phys.: Conf. Ser. 117, 012018 (2008).

${ }^{7}$ B. Reynard and R. Caracas, Chem. Geol. 262, 159 (2009).

${ }^{8}$ K. Hermansson, M. M. Probst, G. Gajewski, and P. D. Mitev, J. Chem. Phys. 131, 244517 (2009).

${ }^{9}$ P. V. Dunmore, J. Chem. Phys. 57, 3348 (1972).

${ }^{10}$ A. P. J. Jansen, W. J. Briels, and A. Van der Avoird, J. Chem. Phys. 81, 3648 (1984).

${ }^{11}$ W. J. Briels, A. P. J. Jansen, and A. Van der Avoird, Adv. Quantum Chem. 18, 131 (1986).

${ }^{12}$ J. C. Raich and R. D. Etters, Phys. Rev. 168, 425 (1968).

${ }^{13}$ F. G. Mertens and W. Biem, Z. Phys. 250, 273 (1972).

${ }^{14}$ W. J. Briels, A. P. J. Jansen, and A. Van der Avoird, J. Chem. Phys. 81, 4118 (1984).

${ }^{15}$ H. M. James and T. A. Keenan, J. Chem. Phys. 31, 12 (1959).

${ }^{16}$ S. J. Clark, M. D. Segall, C. J. Pickard, P. J. Hasnip, M. I. J. Probert, K. Refson, and M. C. Payne, Z. Kristallogr. 220, 567 (2005).

${ }^{17}$ The details of the CASTEP LD calculations are the following. Pseudopotentials were taken from the simulation package, to be precise, the oxygen (O_02.recpot), hydrogen (H_04.recpot), and magnesium (Mg_OP1.recpot) PPs were chosen. The plane-wave cutoff was set to $1100 \mathrm{eV}$ and an $8 \times 8 \times 6 \Gamma$-centered grid of k-points was used for the electronic sampling. Finite-basis set corrections (Ref. 26) were applied during the geometry optimization. A $7 \times 7 \times 5$ grid was used for the phonon sampling, and dispersion curves were calculated from a Fourier interpolation of the dynamical matrix (Refs. 27 and 28) in conjunction with the cumulant sum method of Parlinski (Ref. 29) to construct the aperiodic force constant matrix. The largest error due to the interpolation, throughout the whole Brillouin zone including all modes, was estimated to $\sim 3 \mathrm{~cm}^{-1}$ and the mean error of $\sim 0.5 \mathrm{~cm}^{-1}$.

${ }^{18}$ G. Kresse, J. Hafner, Phys. Rev. B 47, 558 (1993); 49, 14251 (1994).

${ }^{19}$ G. Kresse and J. Furthmüller, Phys. Rev. B 54, 11169 (1996).

${ }^{20}$ G. Kresse and J. Furthmüller, Comput. Mater. Sci. 6, 15 (1996).

${ }^{21}$ B. S. Chakoumakos, C.-K. Loong, and A. J. Schultz, J. Phys. Chem. B 101, 9458 (1997).

${ }^{22}$ T. S. Duffy C. Meade, H.-K. Fei, Y. Mao, and R. J. Hemley, Am. Mineral. 80, 22 (1995).

${ }^{23}$ E. F. de Oliveira and Y. Hase, Vib. Spectrosc. 25, 53 (2001).

${ }^{24}$ M. Born and K. Huang, Dynamical Theory of Crystal Lattices (Oxford University Press, Oxford, 1954).

${ }^{25}$ W. Cochran and R. A. Cowley, J. Phys. Chem. Solids 23, 447 (1962).

${ }^{26}$ G. P. Francis and M. C. Payne, J. Phys.: Condens. Matter 2, 4395 (1990).

${ }^{27}$ S. Baroni, S. de Gironcoli, and A. Dal Corso, Rev. Mod. Phys. 73, 515 (2001).

${ }^{28}$ X. Gonze and C. Lee, Phys. Rev. B 55, 10355 (1997).

${ }^{29}$ K. Parlinski, Z. Q. Li, and Y. Kawazoe, Phys. Rev. Lett. 78, 4063 (1997). 